\title{
Phosphorylation of NtMAP65-1 by a MAP kinase down-regulates its activity of microtubule bundling and stimulates progression of cytokinesis of tobacco cells
}

\author{
Michiko Sasabe, ${ }^{1}$ Takashi Soyano, ${ }^{1,3}$ Yuji Takahashi, ${ }^{1}$ Seiji Sonobe, ${ }^{2}$ Hisako Igarashi, ${ }^{2,4}$ \\ Tomohiko J. Itoh, ${ }^{1}$ Mikiko Hidaka, ${ }^{1}$ and Yasunori Machida ${ }^{1,5}$ \\ ${ }^{1}$ Division of Biological Science, Graduate School of Science, Nagoya University, Chikusa-ku, Nagoya 464-8602, Japan \\ ${ }^{2}$ Graduate School of Life Science, University of Hyogo, Hyogo 678-1297, Japan
}

\begin{abstract}
The tobacco mitogen-activated protein kinase (MAPK) cascade, which includes MAPK NRK1/NTF6, positively regulates expansion of the cytokinetic machinery known as the phragmoplast, which is followed by the synthesis of cell plates for completion of cell division. However, molecular events lying between the MAPK and phragmoplast expansion were not known. Here, we show that NRK1/NTF6 phosphorylates the threonine residue at position 579 in NtMAP65-1a, a microtubule-associated (MT-associated) protein. Levels of phosphorylated NtMAP65-1 increase during late $M$ phase of the cell cycle, when NRK1/NTF6 is activated. Phosphorylated NtMAP65-1 is concentrated at the equator of phragmoplast, as is NRK1/NTF6. Overexpression of mutant forms of NtMAP65-1a that cannot be phosphorylated by NRK1 delays progression of the M phase and phragmoplast expansion, also rendering phragmoplast structures resistant to an MT-depolymerizing drug. Phosphorylation of NtMAP65-1 by NRK1/NTF6 down-regulates its MT-bundling activity in vitro. These results suggest that phosphorylation of NtMAP65-1 by NRK1/NTF6 also reduces its MT-bundling activity in vivo, which enhances destabilization and turnover of MTs at the phragmoplast equator, perhaps facilitating phragmoplast expansion.
\end{abstract}

[Keywords: MAP65/PRC1; MAPK; cytokinesis; microtubule; microtubule-associated proteins; phragmoplast] Supplemental material is available at http://www.genesdev.org.

Received January 6, 2006; revised version accepted February 21, 2006.

Cell division in eukaryotes requires dynamic changes in cytoskeletal structures that consist of microtubules (MTs) and microfilaments (Nishihama and Machida 2001; Jürgens 2005). During cytokinesis, the final critical step in the cell division, cells form a cytokinesis-specific apparatus (known as the central spindle in animals and the phragmoplast in plants) that plays a key role in cytokinesis (Field et al. 1999; Nishihama and Machida 2001). These structures develop from late anaphase to telophase between the two daughter nuclei, and consist of two bundles of antiparallel MTs. As cytokinesis proceeds, the central spindle becomes compacted in animal

Present addresses: ${ }^{3}$ Laboratory of Plant Molecular Biology, The Rockefeller University, 1230 York Avenue, New York, NY 10021, USA, ${ }^{4}$ RIKEN Plant Science Center, Yokohama, Kanagawa 230-0045, Japan. ${ }^{5}$ Corresponding author.

E-MAIL yas@biol1.bio.nagoya-u.ac.jp; FAX 81-52-789-2966.

Article published online ahead of print. Article and publication date are at http://www.genesdev.org/cgi/doi/10.1101/gad.1408106. cells and the phragmoplast expands centrifugally in plant cells, and new membranes and/or cell walls are generated inside or outside the midzone of the central spindle or the phragmoplast to separate the two daughter cells from each other (Otegui et al. 2005). These dynamic processes appear to be mediated by the turnover of MTs, which involves the depolymerization of MTs and the polymerization of tubulin at the plus ends (Shelden and Wadsworth 1990; Asada et al. 1991; Hush et al. 1994; Straight and Field 2000). Recently, Austin et al. (2005) have reported that somatic-type phragmoplast MTs do not interdigitate at the cell plate mid-line and that distinct MT plus-end geometries are seen during the different stages of cytokinesis.

The members of the MAP65/Ase1/PRC1 family (designated the MAP65 family herein)-including plant MAP65, yeast Ase1, and human PRC1-participate in the bundling of MTs (Chang-Jie and Sonobe 1993; Chan et al. 1999; Mollinari et al. 2002; Schuyler et al. 2003; 
Smertenko et al. 2004; Van Damme et al. 2004b). Members of this family are concentrated at the midzone of the central spindle and the phragmoplast during cytokinesis, and their appropriate organization seems to be required for completion of cytokinesis (Pellman et al. 1995; Juang et al. 1997; Jiang et al. 1998; Mollinari et al. 2002; Schuyler et al. 2003; Müller et al. 2004; Verbrugghe and White 2004; Vernì et al. 2004). PRC1 is phosphorylated by cyclin-dependent protein kinases (CDKs), and overexpression of a mutant form of PRC1 that cannot be phosphorylated by CDKs results in the bundling of the MTs of the metaphase spindle and interruption of the progression from metaphase to anaphase, suggesting that phosphorylation of PRC1 by CDKs is required for the metaphase-to-anaphase transition (Mollinari et al. 2002). Phosphorylation of AtMAP65-1 by CDKs might also regulate the targeting of MAP65 to phragmoplast MTs (Mao et al. 2005). However, the ways in which MAP65 proteins control cytokinetic processes, after the inactivation of CDKs at the metaphase-to-anaphase transition, remain to be characterized.

The NACK-PQR pathway, a mitogen-activated protein kinase (MAPK) cascade that is required for plant cytokinesis, is activated during late $\mathrm{M}$ phase and conserved in both tobacco and Arabidopsis. This pathway consists of NACK1, NACK2, kinesin-like proteins (KLPs), NPK1 (nucleus- and phragmoplast-localized protein kinase 1) MAPK kinase kinase (MAPKKK), NQK1/ NtMEK1 MAPK kinase (MAPKK), and NRK1/NTF6 MAPK in tobacco, and NPK1 is activated by direct binding to NACK1 (Nishihama et al. 2001, 2002; Ishikawa et al. 2002; Soyano et al. 2003). During cytokinesis, NACK1, NPK1, NQK1/NtMEK1, and NRK1/NTF6 are found at the equatorial zone of the phragmoplast (Calderini et al. 1998; Nishihama et al. 2001, 2002; our unpublished data). Our recent studies have demonstrated that the NACK-PQR pathway is required for cytokinesis and, in particular, for the expansion of the phragmoplast, and its functions appear to be related to the stability of phragmoplast MTs (Nishihama et al. 2001, 2002; Ishikawa et al. 2002; Soyano et al. 2003).

AtNACK1/HINKEL, ANP1 (ANP2 and ANP3), and ANQ1/AtMKK6 genes of Arabidopsis, which are orthologs of the tobacco NACK1, NPK1, and NQK1 genes, respectively, are also required for cytokinesis of Arabidopsis cells (Nishihama et al. 1997, 2002; Krysan et al. 2002; Strompen et al. 2002; Soyano et al. 2003; Tanaka et al. 2004). On the basis of these results, it has been proposed that these factors might control a certain event related to MTs. It is now important to identify factors that act downstream of the NACK-PQR pathway to understand the mechanisms that control cytokinesis.

We report here that NtMAP65-1, a member of the MAP65 family in tobacco, is phosphorylated by NRK1/ NTF6 MAPK and that NRK1-phosphorylated NtMAP65-1 is concentrated at the phragmoplast equator, as is NRK1/ NTF6. Overexpression, in tobacco cells, of a mutant form of NtMAP65-1a that cannot be phosphorylated by NRK1 delayed M-phase progression and expansion of the phragmoplast and increased the stability of MTs, suggesting that phosphorylation of NtMAP65-1 by NRK1/ NTF6 MAPK suppresses the ability of NtMAP65-1 to bundle MTs. Phosphorylation by NRK1 also decreased the MT-bundling activity of NtMAP65-1a in vitro. We propose that the NACK-PQR pathway can enhance destabilization of MTs and MT turnover at the midzone of the phragmoplast via phosphorylation of NtMAP65-1 during cytokinesis, which may positively stimulate phragmoplast expansion.

\section{Results}

\section{NRK1/NTF6 MAPK phosphorylates NtMAP65-1a} in vitro

Tobacco MT-associated proteins (MAPs), which had been partially purified from tobacco suspension-cultured BY-2 cells, were fractionated by anion-exchange column chromatography. Each fraction was subjected to a kinase assay in vitro with or without activated recombinant histidine- and T7-epitope-tagged NRK1 MAPK (His-T7NRK1) in the presence of $\left[\gamma^{-32} \mathrm{P}\right]$ ATP. Two phosphorylated proteins were detected in fractions 24-29 (Fig. 1A). Both proteins were identified as NtMAP65-1a by amino acid sequencing analysis, which suggested that they were derived from the same product of translation.

We performed an immunocomplex kinase assay using recombinant His-T7-NtMAP65-1a as the substrate. BY-2 cells were synchronized at prometaphase by two-step synchronization with aphidicolin and propyzamide, an MT-depolymerizing drug, and the cell cycle was allowed to proceed by removal of propyzamide. As shown in Figure 1B, panel b, the strongest phosphorylation of His-T7NtMAP65-1a was detected in extracts of BY-2 cells prepared $0.5-1 \mathrm{~h}$ after the removal of propyzamide (top panel), namely, at late $M$ phase. The timing of the phosphorylation of NtMAP65-1a by endogenous NRK1/ NTF6 was similar to that of the activation of NPK1 MAPKKK, NQK1/NtMEK1 MAPKK, and NRK1/NTF6 MAPK, as reported previously (Nishihama et al. 2001; Soyano et al. 2003). In contrast to the phosphorylation by NRK1, the strongest phosphorylation by CDKs, which had been prepared using $\mathrm{p} 13^{\text {sucl }}$ beads from the same extracts of BY-2 cells, was observed at $0 \mathrm{~h}$ (prometaphase), and the extent of phosphorylation fell gradually during progression of the cell cycle (Fig. 1B, panel b, middle).

\section{NRK1/NTF6 MAPK phosphorylates a single threonine} residue at position 579 in NtMAP65-1a

We generated several series of truncated NtMAP65-1a proteins and performed kinase assays in vitro with active recombinant NRK1 or CDKs. Only peptides that included the C-terminal fragment of NtMAP65-1a, from residue 352 to residue 580, were phosphorylated by NRK1 and CDKs in vitro (data not shown). There are 


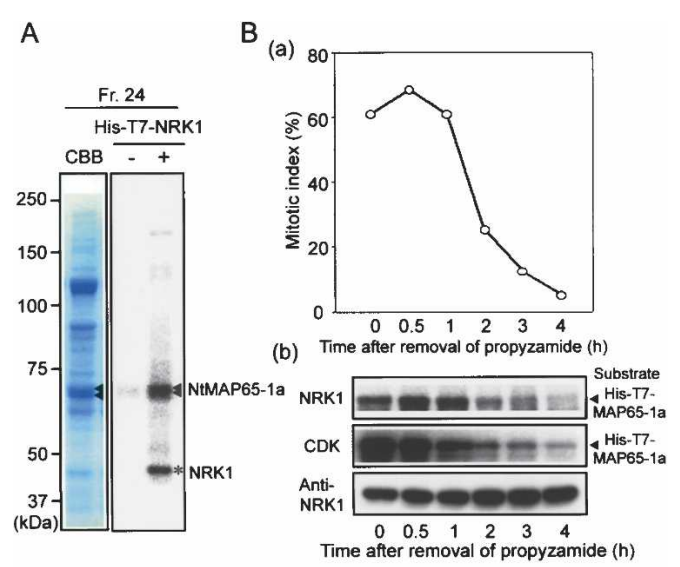

C

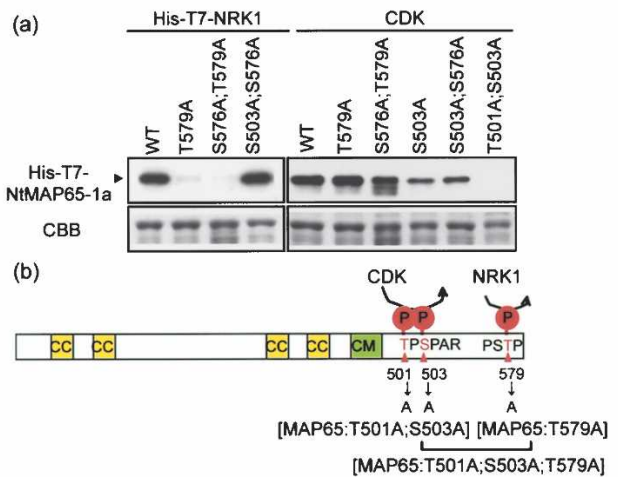

Figure 1. Phosphorylation of NtMAP65-1a by NRK1/NTF6 MAPK in vitro. (A) Phosphorylation in vitro of MAPs by NRK1. MAPs were prepared from tobacco BY-2 cells, and each MAPs fraction was used as substrate in a kinase assay with or without His-T7-NRK1 in the presence of $\left[\boldsymbol{\gamma}^{32} \mathrm{P}\right] \mathrm{ATP}$. (Right) NRK1phosphorylated proteins were detected by autoradiography in fraction 24. (Left) The same fraction was stained with Coomassie Brilliant Blue (CBB). The arrowheads and asterisk show the identified phosphorylated proteins and His-T7-NRK1, respectively. (B) Phosphorylation of NtMAP65-1a by endogenous

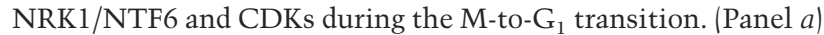
Mitotic indices at the indicated times after removal of propyzamide. (Panel b) Phosphorylation by NRK1/NTF6 and CDKs of His-T7-NtMAP65-1a during the M phase in BY-2 cells. Proteins were extracted from cells that had been harvested at the indicated time after removal of propyzamide. Kinase activities of NRK1/NTF6 (top) and CDKs (middle) were determined by an immunocomplex kinase assay and a $13^{\mathrm{SUCl}}$ beads complex kinase assay, respectively, with His-T7-NtMAP65 as substrate. (Bottom) Proteins extracted from BY-2 cells were also analyzed by immunoblotting with NRK1-specific antibodies (anti-NRK1; (Soyano et al. 2003). (C) Identification of sites of phosphorylation in NtMAP65-1a by NRK1 and CDKs. (Panel a) Kinase assays in vitro using recombinant NRK1 (left) and CDKs prepared from BY-2 cells with p13 ${ }^{\mathrm{SUC} 1}$ beads (right). Recombinant NtMAP65-1a protein and mutant proteins in which serine or threonine residues at positions 501, 503, 576, and/or 579 have been replaced by alanine in various combinations were used as substrates. (Panel b) A schematic representation of NtMAP651a. The coiled-coil motif $(\mathrm{CC})$ and the motif $(\mathrm{CM})$ that is conserved in the MAP65 family are shown as yellow and green boxes, respectively. Sites of phosphorylation $(P)$ by NRK1 and CDKs and the amino acid substitutions in the various mutant forms of NtMAP65-1a are indicated. four potential target sequences for phosphorylation (S/ TP) by MAPK and CDKs in this fragment (threonine residues at positions 501 and 579 and serine residues at positions 503 and 576, designated Thr-501, Thr-579, Ser503, and Ser-576, respectively). We generated mutant NtMAP65-1a proteins, in which these residues were replaced by alanine residues in various combinations, and examined whether these mutants were phosphorylated by NRK1 or CDKs in vitro. The recombinant NtMAP65la proteins were phosphorylated efficiently by NRK1, and all of the proteins were estimated to be phosphorylated under conditions we used (data not shown). The protein with a mutation at Thr-579 (MAP65:T579A) and the doubly mutated protein with mutations at Thr-501 and Ser-503 (MAP65:T501A;S503A) in His-T7NtMAP65-1a were not phosphorylated by NRK1 and by CDKs, respectively (Fig. 1C, panel a). These results demonstrated that NtMAP65-1a has a single site for phosphorylation by NRK1/NTF6 MAPK-namely, Thr-579and that Thr-501 and Ser-503 can be phosphorylated by CDKs (Fig. 1C, panel b).

\section{Phosphorylation of Thr-579 of NtMAP65-1 in vivo}

We prepared antibodies against NtMAP65-1a that had been phosphorylated at Thr-579 (anti-pT579MAP65) and nonphosphorylated NtMAP65-1a (anti-MAP65). AntipT759MAP65 detected only NRK1-phosphorylated HisT7-NtMAP65-1a, and anti-MAP65 antibodies detected both phosphorylated and nonphosphorylated His-T7NtMAP65-1a proteins (Fig. 2A, panel a). CDK-phosphorylated His-T7-NtMAP65-1a was not recognized by anti-pT579MAP65 (data not shown). Tobacco has at least three members of the MAP65-1 subfamily (namely, NtMAP65-1a, NtMAP65-1b, and NtMAP65-1c), which are $>85 \%$ identical in terms of amino acid sequences to one another (Hussey et al. 2002). Our anti-pT579MAP65 recognized all three NRK1-phosphorylated tobacco proteins to similar extents (Supplementary Fig. 1), but not NRK1-phosphorylated AtMAP65-1, AtMAP65-2, and AtMAP65-3, which have putative MAPK-phosphorylation site (data not shown). In addition, antipT579MAP65 detected a single protein of $\sim 65 \mathrm{kDa}$ in extracts of BY-2 cells, the estimated molecular mass of NtMAP65-1a, NtMAP65-1b, and NtMAP65-1c (Fig. 2A, panel b). It appears that anti-pT579MAP65 is specific for phosphorylated forms of the NtMAP65-1 subfamily in tobacco.

We examined the phosphorylation of NtMAP65-1 at Thr-579 during the $M$ phase using our antibodies. We prepared protein extracts of BY-2 cells that had been synchronized by the two-step procedure with aphidicolin and propyzamide, and subjected them to Western blotting analysis with anti-pT579MAP65 and anti-MAP65. Both antibodies recognized a single protein of $65 \mathrm{kDa}$ (Fig. 2A, panel b, middle and bottom). After removal of propyzamide, the level of NtMAP65-1- $\mathrm{P}_{579}$ rose from 0.5 to $1 \mathrm{~h}$ and fell to the background level at $4 \mathrm{~h}$ (Fig. 2A, panel b, bottom), although the level of NtMAP65-1 was invariable (Fig. 2A, panel b, middle). This increase in 
A

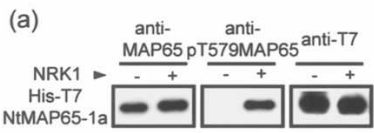

(b)
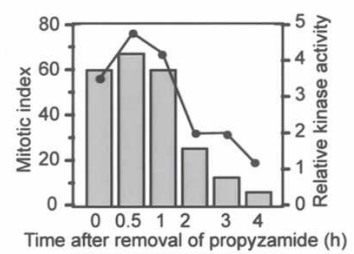

100

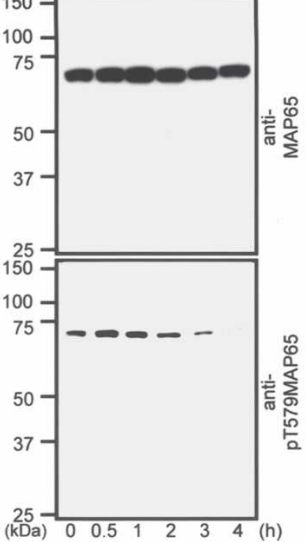

B

(a)

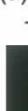

Tubulin

anti-MAP65

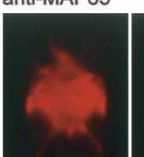

DAPI
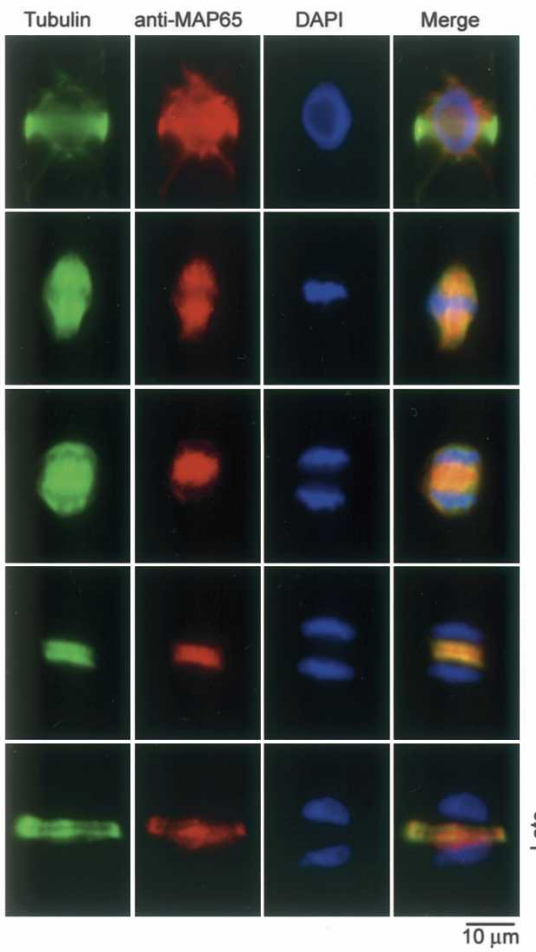

Figure 2. Phosphorylation of NtMAP65-1 at Thr-579 and subcellular localization of NRK1-phosphorylated NtMAP65-1 in tobacco BY-2 cells. (A) Phosphorylation of Thr-579 in NtMAP65-1a during M phase. (Panel a) Specificity of antibodies against pT579NtMAP65. (Panel b) Accumulation of NtMAP65-1 and NtMAP65-1-P ${ }_{579}$ during the M phase. (Top) Mitotic indices are represented as bars, and the activity of NRK1 is plotted as filled circles. Proteins were extracted from BY-2 cells that had been harvested at the indicated times after removal of propyzamide, and immunoblotting analysis was performed with anti-MAP65 (middle) or anti-pT579MAP65 (bottom). (B) Subcellular localization of NtMAP65-1 and NtMAP65-1-P ${ }_{579}$ in BY-2 cells. (Panel $a$ ) Localization of NtMAP65-1. (Panel $b$ ) Localization of NtMAP65-1-P ${ }_{579}$. BY-2 cells at various phases of the cell cycle were stained with anti-MAP65 (panel $a_{\text {; }}$ red), anti-pT579MAP65 (panel $b$; red), tubulin-specific antibodies (green), and 4'6-diamidino-2-phenylindole (DAPI), which is specific for nuclei (blue). Merged images are shown on the right.

phosphorylation was consistent with the activation of endogeneous NRK1 MAPK (Fig. 2A, panel b, top).

We examined the subcellular localization of NtMAP65-1 and of NtMAP65-1-P 579 during the M phase by immunostaining (Fig. 2B). Anti-MAP65 bound to all cytoskeletal structures throughout mitosis, including preprophase bands, spindles, and phragmoplasts (Fig. 2B, panel a). Experiments with green fluorescent protein-fused NtMAP65-1a (GFP-NtMAP65-1a) yielded essentially the same results (data not shown). Patterns of signals due to anti-pT579MAP65 were basically similar to those due to anti-MAP65 from interphase to metaphase (Fig. 2B, panel b). Signals were, however, concentrated in the midzone of early phragmoplasts at anaphase (Fig. 2B, panel $b$; anaphase). From telophase to late telophase, signals were localized at the phragmoplast equator (Fig. 2B, panel b; telophase and late telophase). Thus, NtMAP65-1-P 579 was concentrated at the equatorial region of the phragmoplast during its lateral expansion, consistent with localization of components in the NACK-PQR cascade (Calderini et al. 1998; Bögre et al. 1999; Nishihama et al. 2001; Nishihama et al. 2002; our unpublished data).
Expression of MAPK-nonphosphorylatable NtMAP65-1a delays the cell cycle

We postulated that overexpression of NRK1-nonphosphorylatable NtMAP65-1a might affect cell division by dominant-negative interference with the activity of Thr579-phosphorylated NtMAP65-1a. We transformed BY-2 cells with cDNA constructs for GFP-fused NtMAP65-1a (MAP65:WT) and various mutants of NtMAP65-1a, under the control of a dexamethasone-inducible (DEX-inducible) transcription system (Aoyama and Chua 1997). In MAP65:T579A, MAP65:T501A;S503A, and MAP65: T501A;S503A;T579A, the sites of phosphorylation by NRK1, CDKs, and both kinases were replaced, respectively, by nonphosphorylatable alanine residues (Fig. 1C, panel b).

As shown in Figure 3A, when we synchronized BY-2 cells using aphidicolin, subsequent progression of the cell cycle in cells that expressed the cDNA for MAP65:T579A or the cDNA for MAP65:T501A;S503A; T579A in response to DEX was significantly delayed (Fig. 3A, panels c,e). Peaks of mitotic indices were evident $10-12 \mathrm{~h}$ after removal of aphidicolin. By contrast, 
A
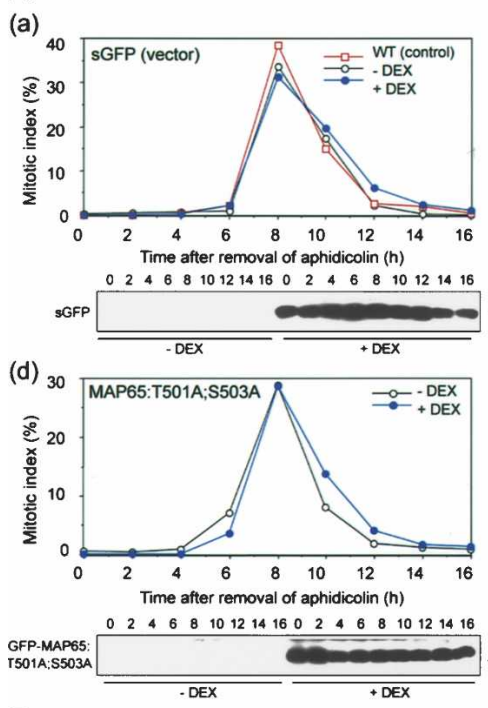

B (a)
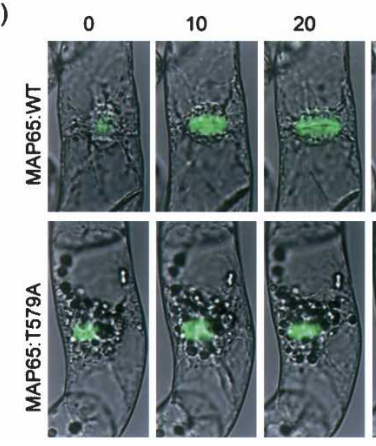

(b)

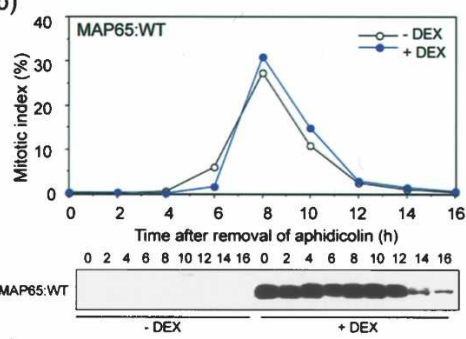

(e) 30 MAP65:T501A;5503A;T579A

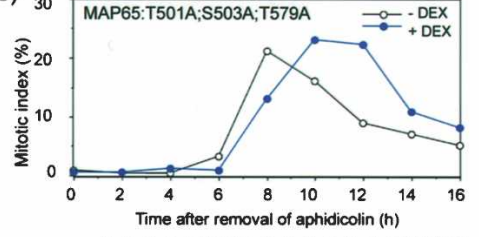

\begin{tabular}{c} 
GFP-MAPG5: \\
T501A:SSOSA:T579A \\
\hline
\end{tabular} 024681012141802466810121416
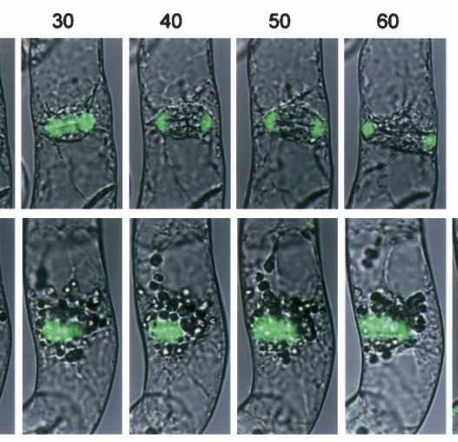

90

(min)

(c)

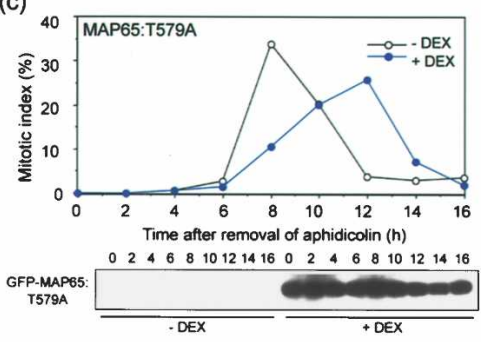

(b)

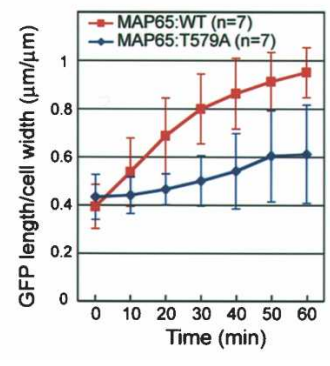

Figure 3. Retardation of progression through the M phase and phragmoplast expansion upon overexpression of NRK1-nonphosphorylatable NtMAP65-1a. (A) Progression of the cell cycle in BY-2 cells that expressed GFP-fused NtMAP65-1a and GFP-fused derivatives of NtMAP65-1a with mutations at sites of phosphorylation by NRK1 and/or CDKs. Fusion constructs were transcribed under control of a DEX-inducible transcription system. BY-2 cells that expressed GFP (panel $a$ ), GFP-MAP65:WT (panel $b$ ), GFP-MAP65:T579A (panel $c$ ), GFP-MAP65:T501A;S503A (panel $d$ ), and GFP-MAP65:T501A;S503A;T579A (panel $e$ ) were treated with aphidicolin for synchronization. DEX $(0.5 \mu \mathrm{M})$ was added at $12 \mathrm{~h}$ after the start of treatment with aphidicolin (+DEX). After incubation for another $12 \mathrm{~h}$, cells were subcultured in fresh medium with $0.5 \mu \mathrm{M} \mathrm{DEX}$, and mitotic indices were plotted at indicated times after removal of aphidicolin. Control experiments were performed, in parallel, in the absence of DEX (-DEX). (Panels $a-e$, bottom) Levels of fusion proteins were examined by immunoblotting with GFP antibodies. (B) Retardation of the lateral expansion of the phragmoplast by NRK1-nonphosphorylatable NtMAP65-1a. (Panel a) Time-lapse micrographs of phragmoplasts that harbored GFP-fused MAP65:WT (top) and GFP-fused MAP65:T579A (bottom) in living BY-2 cells. Confocal micrographs were recorded at indicated times after the start of observations of phragmoplast formation. (Panel $b$ ) The ratio of the length of the fluorescent region to the cell width was plotted against time. Cells were selected randomly. Values are mean \pm S.E.M. $(n=7)$.

the cell cycle of cells that expressed the vector cDNA, the cDNA for MAP65:WT, or the cDNA for MAP65: T501A;S503A with mutations at CDK-phosphorylation sites was normal, with peaks of mitotic indices at $8 \mathrm{~h}$ (Fig. 3A, panels a,b,d). The delay seemed to be caused by a reduced rate of progression of the $\mathrm{M}$ phase rather than a delay in the initiation of this phase because there were no differences in timing of the entry into $M$ phase $6 \mathrm{~h}$ after the removal of aphidicolin (Fig. 3A). Analysis by flow cytometry showed that the timing of progression from the $S$ phase to $G_{2}$ was similar in all cell lines (data not shown).

We monitored expansion of phragmoplast in cells that harbored the GFP-fused MAP65:WT or GFP-fused MAP65:T579A construct (Fig. 3B). In the presence of
DEX, MAP65:WT and MAP65:T579A were found similarly on the phragmoplast, and so we were able to monitor expansion of the phragmoplast in terms of fluorescence derived from GFP-fused MAP65:WT and GFPfused MAP65:T579A. In BY-2 cells that expressed MAP65:WT, most phragmoplasts were fully expanded within $60 \mathrm{~min}$ after the initiation of phragmoplast formation (Fig. 3B, panel a, top). By contrast, a significant proportion of BY-2 cells that expressed MAP65:T579A had different profiles. Although phragmoplasts were formed, many failed to reach the edges of cells within 90 min (Fig. 3B, panel a, bottom). Ratios of the "length" of the fluorescent region to the width of the cell revealed clearly that expansion of the fluorescent region in MAP65:T579A cells was slower than that in MAP65:WT 
cells (Fig. 3B, panel b). Thus, overexpression of NRK1nonphosphorylatable NtMAP65-1a delayed expansion of the phragmoplast, suggesting that the low rate of phragmoplast expansion was responsible, at least in part, for the delayed progression of the $M$ phase described above. The similar delayed expansion is observed when cDNAs for dominant-negative mutants of NACK1, NPK1, and NQK1 were overproduced (Nishihama et al. 2001, 2002; Soyano et al. 2003).

Phosphorylation of NtMAP65-1a at Thr-579 destabilizes the organization of MTs in vivo

We examined whether phosphorylation of NtMAP65-1a by NRK1/NTF6 MAPK might affect MT arrays in vivo. We transformed BY-2 cells with cDNAs for various DEXinducible proteins, namely, GFP-MAP65:WT, GFPMAP65:T579A, GFP-MAP65:T501A;S503A, and GFPMAP65:T501A;S503A;T579A, and then we examined the localization of these proteins in transformed cells. Figure 4A shows the fluorescence due to GFP in living transformed cells in the presence of DEX. In the absence of propyzamide, GFP-MAP65 was localized on cortical MTs during interphase, and there were no visible difference between the mutant and the wild-type proteins (Fig. $4 \mathrm{~A}$, top). By contrast, there was a clear difference between cells that expressed GFP-MAP65:T579A (Fig. 4A, lower right panel) and the cells that expressed GFPMAP65:WT (Fig. 4A, lower left panel), in the presence of propyzamide. In cells that expressed GFP-MAP65:WT, signals had diffused into the cytoplasm, while in the cells that expressed GFP-MAP65:T579A, fluorescence due to GFP was detected on conspicuously bundled MTs.

Next, we investigated the stability of MT-containing structures using tubulin-specific antibodies (anti-tubulin). As shown in Figure 4B, cortical MTs and phragmoplasts were disrupted after treatment with $3 \mu \mathrm{M}$ propyzamide in cells that expressed GFP-MAP65:WT or GFPMAP65:T501A;S503A, and the cells became swollen, perhaps because of the disruption of cortical MTs. Moreover, many cells had poorly aligned chromosomes, and the cell cycle appeared to be arrested at prometaphase (Fig. 4B, third row of panels from top, left, and centerright). Cells expressing GFP-MAP65:T579A and GFPMAP65:T501A;S503A;T579A, by contrast, retained well-organized MTs even after treatment with propyzamide, although the integrity of MTs seemed to be altered. At $\mathrm{M}$ phase, some of the cells were multinucleate with an incomplete phragmoplast (Fig. 4B, third and bottom rows of panels, center-left, and right), suggesting that the M phase had somehow progressed in these cells, even in the presence of propyzamide. Although these cells were elongated in the longitudinal direction, no lateral swelling was observed. Disruption of MTs by propyzamide was evident in all cell lines in the absence of DEX (data not shown). Thus, in cells that expressed NRK1-nonphosphorylatable NtMAP65-1a, arrays of at least the cortical MTs and the MTs of the phragmoplast were more resistant to propyzamide than they were in cells that expressed other constructs. These observations suggest that NRK1-nonphosphorylatable NtMAP65-1a enhances the stability of MT arrays in cortical MTs and the phragmoplast in vivo.

Phosphorylation of Thr-579 in NtMAP65-1a reduces its MT-bundling activity in vitro

The His-T7-NtMAP65-1a (MAP65:WT) protein that we prepared from Escherichia coli bound to MTs (data not
A

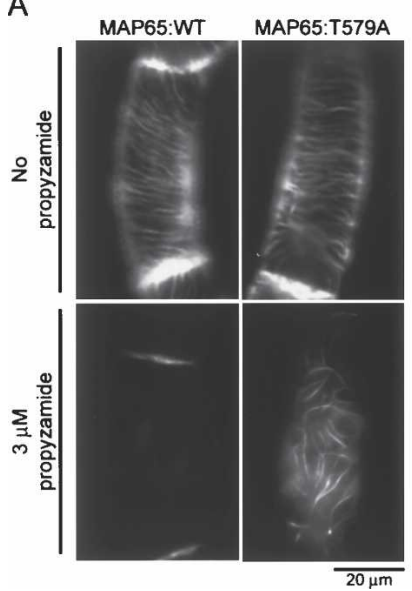

B
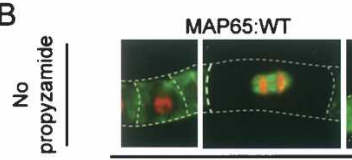

MAP65:T579A
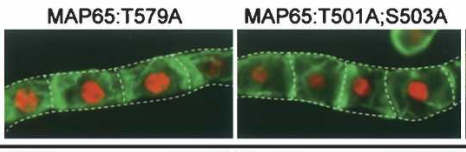

MAP65
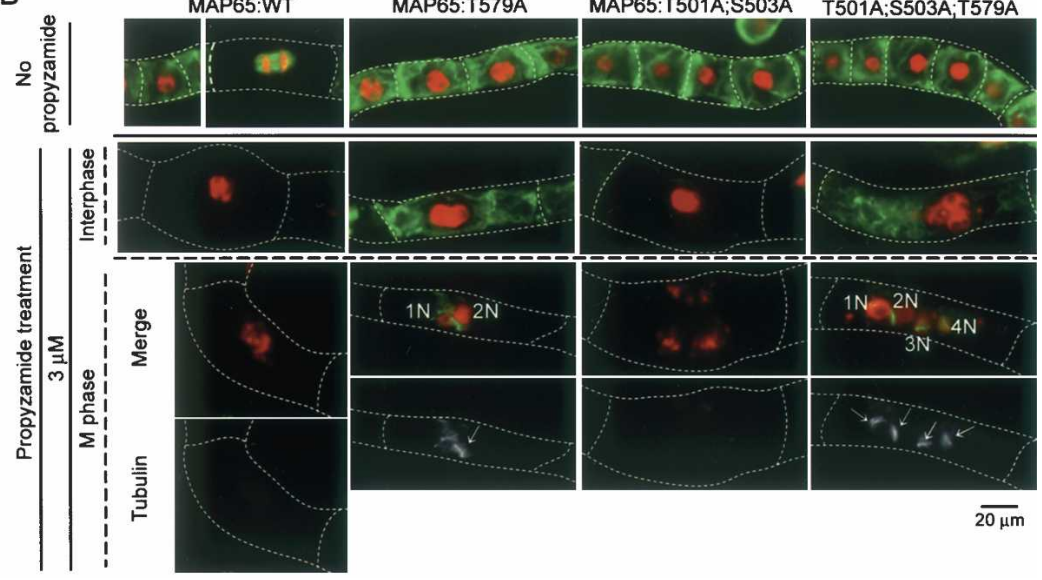

Figure 4. Effects of overexpression of NRK1-nonphosphorylatable NtMAP65-1a on the response to propyzamide of cortical and phragmoplast MTs. (A) Localization of GFP-NtMAP65-1a (MAP65:WT) and GFP-NtMAP65-1a:T579A (MAP65:T579A) in transformed BY-2 cells. Cells were incubated for $12 \mathrm{~h}$ with $0.5 \mu \mathrm{M}$ DEX and then incubated with or without $3 \mu \mathrm{M}$ propyzamide for another $12 \mathrm{~h}$. (B) Distribution of MTs in BY-2 cells that expressed GFP-NtMAP65-1a (MAP65:WT), GFP-NtMAP65-1a:T579A (MAP65:T579A), GFP-NtMAP65-1a:T501A;S503A (MAP65:T501A;S503A), and GFP-NtMAP65-1a:T501A;S503A;T579A (MAP65:T501A;S503A;T579A) in the absence and the presence of propyzamide. Cells were incubated as noted above and then double-stained with mouse antibodies against tubulin (green) and DAPI (for nuclei; red). Bottom panels show images of tubulin (arrows) only to emphasize the stability of MT arrays in cells that expressed the NRK1-nonphosphorylatable protein. (N) Nucleus. 
shown) and bundled MTs in vitro (Fig. 5A). We examined the effects of the phosphorylation of NtMAP65-1a by NRK1 and/or CDKs on its bundling activity. When NRK1-phosphorylated MAP65:WT was added at concentrations of $0.1 \mu \mathrm{M}$ and lower, the bundling of MTs was less prominent than it was when nonphosphorylated MAP65:WT was added at the same respective concentrations (Fig. 5A). When both types of NtMAP65-1a protein were added at $0.5 \mu \mathrm{M}$, no difference was observed between their effects.

To quantitate the relative efficiencies of MT bundling by NtMAP65-1a proteins, we performed a cosedimentation assay with low-speed centrifugation, anticipating that nonbundled MTs and bundled MTs would be recovered in the supernatant and pellet, respectively. We subjected such supernatants and pellets to SDS-PAGE, and stained gels with Coomassie brilliant blue (Fig. 5B, panel a). Then we calculated relative levels of tubulin in pellet fractions and plotted our results (Fig. 5B, panel b). Under our conditions, most of the tubulin was recovered in the supernatant fraction in the absence of NtMAP65, while most of the tubulin was recovered in the pellet fraction in the presence of $0.3 \mu \mathrm{M}$ MAP65:WT (Fig. 5B, panel a, lanes 1,2 ).

As shown in Figure 5B, NRK1-phosphorylated proteins were less-efficient mediators of MT bundling at concentrations of $0.15 \mu \mathrm{M}$ and lower than nonphosphorylated NtMAP65-1a. In contrast to phosphorylation by NRK1, phosphorylation of NtMAP65-1a by CDKs only slightly suppressed the bundling of MTs by NtMAP65-1a (Fig. 5B). When NtMAP65-1a was phosphorylated by both NRK1 and CDKs together, there was no synergistic effect on the suppression of MT bundling (Fig. 5B). When we treated NRK1-nonphosphorylatable NtMAP65-1a (MAP65:T579A) with NRK1, its ability to bundle MTs was the same as that of NtMAP65:T579A without treatment with NRK1 (Fig. 5B, panel a, lanes 9-12). These results showed that the decrease in the MT-bundling activity of NtMAP65-1a in vitro was mainly due to phosphorylation by NRK1 MAPK. Note that the phosphorylation of NtMAP65-1a by NRK1 and by CDKs did not affect the ability of NtMAP65-1a to bind to MTs /data not shown).

\section{Discussion}

The present study has demonstrated that tobacco NRK1/ NTF6 MAPK phosphorylates NtMAP65-1, and that such phosphorylation down-regulates the MT-bundling activity of NtMAP65-1 and stimulates progression of cytokinesis of tobacco cells. Thus, we linked the NACK-PQR MAPK pathway to phragmoplast expansion through the regulatory function of NtMAP65-1.

\section{Phosphorylation of Thr-579 in NtMAP65-1a by NRK1/NTF6 MAPK is a key reaction in the control of cytokinesis}

We showed here that NRK1/NTF6 MAPK phosphorylates NtMAP65-1a directly at Thr-579 in vitro (Fig. 1). We have also provided evidence for such phosphorylation in vivo, as follows: (1) The level of the phosphorylated members of the NtMAP65-1 subfamily, as detected with phospho-Thr-579-specific antibodies, increased at late $M$ phase, when NRK1/NTF6 MAPK is activated (Fig.
Figure 5. Suppression of the MT-bundling activity in vitro of NtMAP65-1a upon phosphorylation by NRK1/ NTF6 MAPK. (A) Dark-field micrographs of bundled MTs after bundling had been promoted by His-T7NtMAP65-1a (MAP65:WT). Taxol-stabilized MTs were incubated with MAP65:WT (top) or NRK1-phosphorylated MAP65:WT (middle) at the indicated concentrations. The bottom panel shows the distribution of MTs in the absence of NtMAP65-1a. (B) Quantification of bundled MTs promoted by NtMAP65-1a. (Panel $a$ ) Staining by Coomassie Brilliant Blue of tubulin in bundled and unbundled MTs after SDS-PAGE. Bundled MTs with His-T7-NtMAP65-1a and individual MTs were separated by low-speed centrifugation. Aliquots of supernatants $(S)$, which included nonbundled MTs and pellets $(P)$ that included bundled MTs, were subjected to SDS-PAGE and then the gel was stained. (Panel $b$ ) Graphs showing ratios of bundled MTs to total MTs at various concentrations of His-T7-NtMAP65-1a (MAP65:WT). Relative amounts of MTs that had been bundled by MAP65:WT (open squares), NRK1-phosphorylated proteins (red filled squares), CDK-phosphorylated proteins (open diamonds), and NRK1 plus CDK-phosphorylated proteins (blue filled diamonds) were calculated from the results of cosedimentation assays after low-speed centrifugation. Independent assays were performed more than three times, and average values with standard deviations are shown.
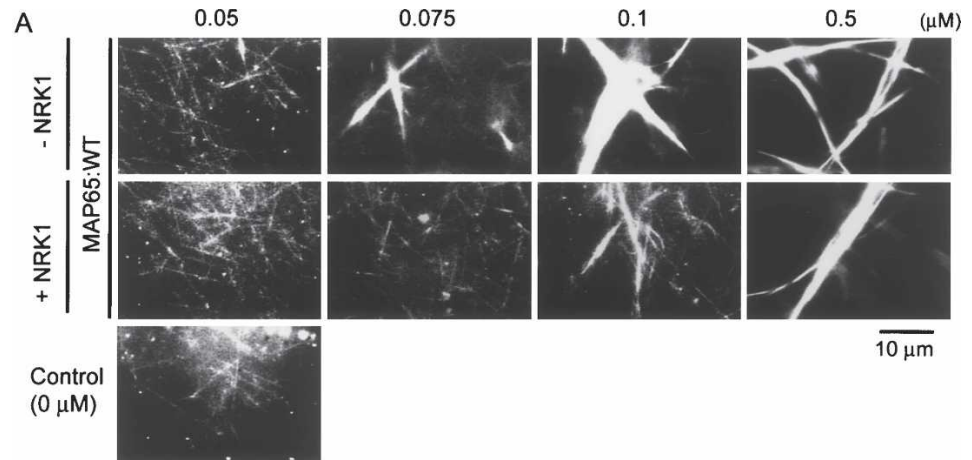

B (a)
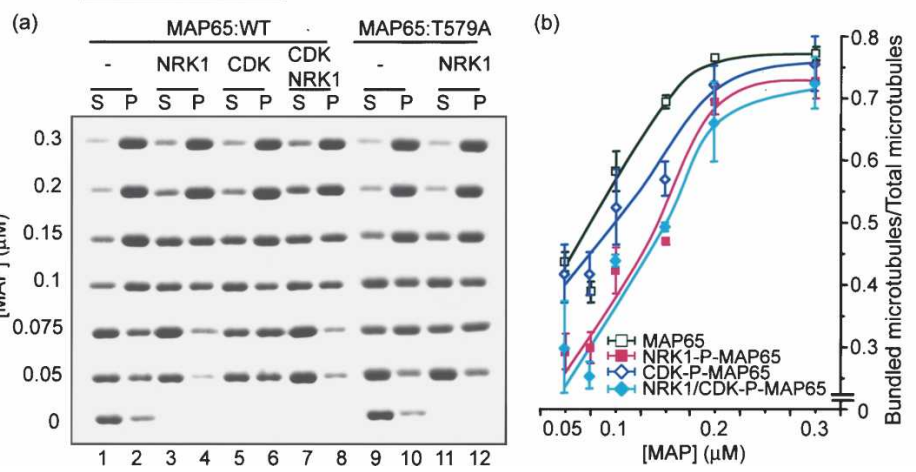
2A). (2) Phosphorylated forms of NtMAP65-1 were concentrated at the equatorial region of phragmoplasts, at which NRK1/NTF6 is also concentrated, although nonphosphorylated form(s) were present over the entire phragmoplast (Fig. 2B), as was GFP-fused NtMAP65-1a (Sasabe et al. 2006). (3) Overexpression of NRK1-nonphosphorylatable mutants of NtMAP65-1a delayed progression of the $M$ phase and expansion of the phragmoplast (Fig. 3). Such mutant NtMAP65-1a might have had a dominant inhibitory effect on the actions of endogenous phosphorylated MAP65-1a and/or other members of the MAP65-1 subfamily, because they bound to MTs in vivo and in vitro as avidly as the wild-type protein and were distributed similarly to the wild-type protein in BY-2 cells (our unpublished data). We showed previously that dominant-negative mutations in components of this MAPK cascade-namely, NACK1, NPK1, and NQK1inhibited expansion of the phragmoplast (Nishihama et al. 2001, 2002; Soyano et al. 2003). Therefore, the results of our experiments in vivo suggest that Thr-579 of NtMAP65-1a is phosphorylated in vivo by NRK1/NTF6 MAPK, which is localized at the phragmoplast equator and positively regulates progression of the $M$ phase and cytokinesis.

Members of the MAP65 protein family are required for cytokinesis in human, Drosophila, and Arabidopsis (Jiang et al. 1998; Mollinari et al. 2002; Müller et al. 2004; Vernì et al. 2004). Thus, our data suggest that phosphorylation of Thr-579 in NtMAP65-1 by NRK1/NTF6 MAPK is a key reaction that controls the functions of NtMAP65-1a during cytokinesis. Various members of the MAP65 family are concentrated at the phragmoplast in a variety of plants (Smertenko et al. 2000, 2004; Müller et al. 2004; Van Damme et al. 2004a). Both AtMAP65-1 and AtMAP65-2 of Arabidopsis, which are the most strongly homologous to members of the tobacco NtMAP65-1 family among MAP65 isotypes, and AtMAP65-3 are also phosphorylated by NRK1/NTF6 (our unpublished data). It will be of interest to examine whether the functions of other MAP65 members are also regulated by NRK1/NTF6 or other MAPKs.

It is noteworthy that Thr-579-phosphorylated NtMAP65 was consistently detected throughout the cell cycle in BY-2 cells, even though NRK1/NTF6 is activated only at late $M$ phase (Fig. 2B; our unpublished data). Member(s) of the MAP65-1 subfamily might also be phosphorylated by as yet unidentified MAPKs. Overexpression of the NRK1-nonphosphorylatable mutant of MAP65-1a, however, only affected progression of the $M$ phase and cytokinesis (Fig. 3). Phosphorylation of Thr-579 of NtMAP65-1 by NRK1/NTF6 MAPK seems to play a more critical role during the $M$ phase than that during other phases.

Overexpression of CDK-nonphosphorylatable mutants of NtMAP65-1a does not affect the progression of the cell cycle

Recombinant NtMAP65-1a protein was phosphorylated in vitro by CDKs prepared from BY-2 cells at M phase
(Fig. 1). However, overexpression of CDK-nonphosphorylatable mutants of NtMAP65-1a did not affect the progression of the cell cycle at any phase (Fig. 3) nor did it affect MT-bundling activity (Fig. 5B). Our results are consistent with those obtained with AtMAP65-1 by Mao et al. (2005). By contrast, overexpression of CDK-nonphosphorylatable PRC1 (a mammalian MAP65 homolog) in human cells resulted in cell cycle arrest (Mollinari et al. 2002), indicating that progression of the cell cycle requires phosphorylation of PRC1 by CDK. As demonstrated in the present study, the control of MAP65 activity by NRK1/NTF6 MAPK appears to be crucial for plant cytokinesis, perhaps because expansion of the phragmoplast, which consists of huge bundles of MTs, must occur after the inactivation of CDKs at metaphase. It must be interesting to investigate the involvement of MAPKs in the regulation of MAP65 family in cytokinesis of animal cells.

MAPK might accelerate expansion of the phragmoplast via phosphorylation of NtMAP65-1 during cytokinesis

As discussed above, phosphorylation in vivo of Thr-579 of NtMAP65-1 by NRK1/NTF6 seems to enhance progression of the $M$ phase. What molecular events might be regulated by such phosphorylation in vivo? Our results provide some information that might help to answer this question. Members of the MAP65 family might play a role in the maintenance of the central spindle in animals (Jiang et al. 1998; Mollinari et al. 2002; Verbrugghe and White 2004; Vernì et al. 2004) and the phragmoplast in plants during cytokinesis (Smertenko et al. 2000; Müller et al. 2004). Some members might also be involved in the bundling of MTs in vivo to stabilize and maintain functional central spindles or phragmoplasts (Fig. 6, left; Mollinari et al. 2002; Müller et al. 2004; Van Damme et al. 2004b; Verbrugghe and White 2004; Vernì et al. 2004). The NRK1-nonphosphorylatable NtMAP651a rendered the phragmoplast and cortical MTs strongly resistant to depolymerization by propyzamide (Fig. 4). In addition, we found that phosphorylation by NRK1/NTF6 decreased the MT-bundling activity of NtMAP65-1 in vitro (Fig. 5). These results suggest that the stability of MTs depends on the MT-bundling activity of MAP65. Therefore, the simplest model that explains the molecular effects of phosphorylation by NRK1/NTF6 MAPK is that such phosphorylation suppresses the bundling activity of NtMAP65-1 in vivo. Phosphorylation of NtMAP65-1 at the phragmoplast midzone might untangle the highly bundled phragmoplast MTs and the resultant relaxed MTs at the phragmoplast midzone might then allow expansion of the phragmoplast, most probably via indirectly enhanced turnover of MTs (Fig. 6, right).

Proteins outside the MAP65 family that are involved in stabilization of the phragmoplast or the central spindle have been identified in plants and animals (Williams et al. 1995; Whittington et al. 2001; Mishima et al. 2002; Twell et al. 2002). In addition, mammalian PRC1 


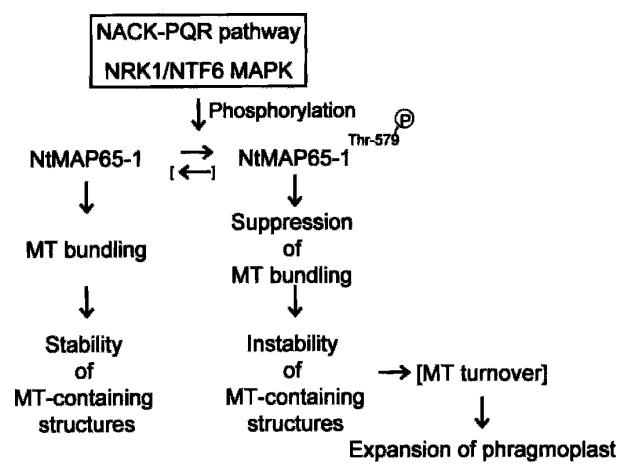

Figure 6. A hypothetical scheme showing the role of the phosphorylation of Thr-579 in NtMAP65-1 during plant cytokinesis. (Left) In this scheme, NtMAP65-1 stabilizes and maintains phragmoplast structures via its strong ability to bundle MTs during cytokinesis. During cytokinesis, in the midzone of the phragmoplast at sites where the components of the NACKPQR pathway, including NRK1/NTF6 MAPK, are activated, NtMAP65-1 is phosphorylated by NRK1/NTF6 at Thr-579, and this phosphorylation results in localized suppression of its MTbundling activity. (Right) Such suppression increases the instability of the organized MT-containing structure and the rate of MT turnover, which results in accelerated expansion of the phragmoplast.

interacts separately with many proteins that are involved in the formation of the midzone of the central spindle (Ban et al. 2004; Kurasawa et al. 2004; Zhu and Jiang 2005), suggesting that regulation of the dynamics of MT-containing structures must be very complex. Further investigations of MAP65-interacting proteins and identification of target proteins, other than MAP65s, in the NACK-PQR pathway are required if we are to understand fully the regulation of cytokinesis by this kinase cascade.

\section{Materials and methods}

\section{Plant materials and transformation}

Tobacco suspension-cultured BY-2 cells were maintained as described elsewhere (Nishihama et al. 2001). Synchronization of the BY-2 cell cycle at the $G_{1} / S$ boundary and two-step synchronization at prometaphase were performed as described previously (Banno et al. 1993; Nishihama et al. 2001). Transformed BY-2 cells were generated by Agrobacterium-mediated transformation (An 1985).

\section{Preparation of MAPs from BY-2 cells}

MAPs were prepared as described by Igarashi et al. (2000). MAPs were fractionated on a Resource Q column (Amersham Bioscience) with a linear gradient of 0 to $1 \mathrm{M} \mathrm{NaCl}$ and desalted with the Microcon YM-10 system (Millipore).

\section{Identification of amino acid sequences and isolation of} cDNA for NtMAP65-1a

Bands of protein were cut from gels after SDS-PAGE. Proteins were digested with lysyl endopeptidase in situ and analyzed by automated protein sequencing. The technique confirmed that the proteins in the chosen bands were identical to NtMAP65-1a. To clone cDNA for NtMAP65-1a, we purified poly(A) $)^{+}$RNAs from BY-2 cells as described elsewhere (Banno et al. 1993). We synthesized cDNA for NtMAP65-1a with specific primers from poly $(\mathrm{A})^{+}$RNAs and cloned the cDNA in pENTR 3C (Invitrogen). Details of the primers are available from the authors upon request.

\section{Site-directed mutagenesis and construction of plasmids}

Mutations in NtMAP65-1a at putative sites of phosphorylation by MAPK and/or CDKs were introduced with a QuikChange Multi Site-Directed Mutagenesis Kit (Stratagene). All reactions were performed according to manufacturer's instructions.

Kinase assays in vitro and immunocomplex and $p 13^{\text {suc } 1}$ beads complex kinase assays

To obtain active NRK1 MAPK, we incubated recombinant HisT7-NRK1 and recombinant GST-NQK in kinase buffer (Nishihama et al. 2001) plus $500 \mu \mathrm{M}$ ATP for $60 \mathrm{~min}$ at $25^{\circ} \mathrm{C}$ and purified on a column of Chelating Sepharose FF (Amersham Biosciences) to eliminate GST-NQK1. Immunocomplex kinase assays were performed as described previously (Nishihama et al. 2001; Soyano et al. 2003; Araki et al. 2004).

Production of antibodies and immunodetection of proteins

Antibodies against NtMAP65-1a (anti-MAP65) and Thr-579phosphorylated NtMAP65-1a (anti-pT579MAP65) were prepared using the synthetic peptides SGSEHSPSTP and SGSEHSPST $\left(\mathrm{PO}_{3} \mathrm{H}_{2}\right) \mathrm{P}$ (residues 571-580 of NtMAP65-1a), respectively, as antigens. Specific antibodies were affinity-purified with the appropriate antigenic oligopeptide (Nishihama et al. 2001). Monoclonal T7 antibodies and GFP antibodies were purchased from Novagen and Clontech, respectively.

Preparation of tubulin from porcine brains for the MT-bundling assay in vitro

Tubulin was purified from porcine brains as described by Itoh et al. (1997). Paclitaxel-stabilized MTs were also prepared and subjected to dark-field microscopy as described by Itoh et al. (1997).

\section{MT-bundling assay in vitro}

NtMAP65-1a or phosphorylated NtMAP65-1a at various concentrations was mixed with $2 \mu \mathrm{M}$ paclitaxel-stabilized MTs. The mixture was incubated for $5 \mathrm{~min}$ at room temperature and then centrifuged at $4500 \times g$ for $5 \mathrm{~min}$ at $25^{\circ} \mathrm{C}$. The resultant supernatants and pellets were subjected to SDS-PAGE. Bands of tubulin were stained with Coomassie brilliant blue, and the relative amounts of tubulin in pellets were calculated with the NIH Image program (http://rsb.info.nih.gov/nih-image), after scanning of gel images as PICT files.

\section{Fluorescence microscopy}

All techniques for microscopic analysis were described previously (Nishihama et al. 2001), with the exception that BY-2 cells were fixed in $3.7 \%$ paraformaldehyde in PM buffer $0.1 \mathrm{M}$ PIPES at pH 6.9, 1 mM EGTA, $0.5 \mathrm{mM} \mathrm{MgSO}_{4}$ ) instead of $3.7 \%$ formaldehyde in sodium phosphate buffer. 


\section{Acknowledgments}

This work was supported in part by a grant from the Program for the Promotion of Basic Research Activities for Innovative Biosciences, by a Grant-in-Aid for Scientific Research on Priority Areas (no. 14036216), and by a Grant-in-Aid for the 21st Century COE Program (System Bioscience) from the Ministry of Education, Culture, Sports, Science and Technology of Japan.

\section{References}

An, G. 1985. High efficiency transformation of cultured tobacco cells. Plant Physiol. 79: 568-570.

Aoyama, T. and Chua, N.H. 1997. A glucocorticoid-mediated transcriptional induction system in transgenic plants. Plant J. 11: 605-612.

Araki, S., Ito, M., Soyano, T., Nishihama, R., and Machida, Y. 2004. Mitotic cyclins stimulate the activity of c-Myb-like factors for transactivation of $\mathrm{G}_{2} / \mathrm{M}$ phase-specific genes in tobacco. J. Biol. Chem. 279: 32979-32988.

Asada, T., Sonobe, S., and Shibaoka, H. 1991. Microtubule translocation in the cytokinetic apparatus of cultured tobacco cells. Nature 350: 238-241.

Austin II, J.R., Segui-Simarro, J.M., and Staehelin, L.A. 2005. Quantitative analysis of changes in spatial distribution and plus-end geometry of microtubules involved in plant-cell cytokinesis. J. Cell Sci. 118: 3895-3903.

Ban, R., Irino, Y., Fukami, K., and Tanaka, H. 2004. Human mitotic spindle-associated protein PRC1 inhibits MgcRacGAP activity toward Cdc42 during the metaphase. I. Biol. Chem. 279: 16394-16402.

Banno, H., Hirano, K., Nakamura, T., Irie, K., Nomoto, S., Matsumoto, K., and Machida, Y. 1993. NPK1, a tobacco gene that encodes a protein with a domain homologous to yeast BCK1, STE11, and Byr2 protein kinases. Mol. Cell. Biol. 13: 4745-4752.

Bögre, L., Calderini, O., Binarova, P., Mattauch, M., Till, S., Kiegerl, S., Jonak, C., Pollaschek, C., Barker, P., Huskisson, N.S., et al. 1999. A MAP kinase is activated late in plant mitosis and becomes localized to the plane of cell division. Plant Cell 11: 101-113.

Calderini, O., Bögre, L., Vicente, O., Binarova, P., Heberle-Bors, E., and Wilson, C. 1998. A cell cycle regulated MAP kinase with a possible role in cytokinesis in tobacco cells. J. Cell Sci. 111: 3091-3100.

Chan, J., Jensen, C.G., Jensen, L.C., Bush, M., and Lloyd, C.W. 1999. The $65 \mathrm{kDa}$ carrot microtubule-associated protein forms regularly arranged filamentous cross-bridges between microtubules. Proc. Natl. Acad. Sci. 96: 14931-14936.

Chang-Jie, J. and Sonobe, S. 1993. Identification and preliminary characterization of a $65-\mathrm{kDa}$ higher-plant microtubule-associated protein. J. Cell Sci. 105: 891-901.

Field, C., Li, R., and Oegema, K. 1999. Cytokinesis in eukaryotes: A mechanistic comparison. Curr. Opin. Cell Biol. 11: 68-80.

Hush, J.M., Wadsworth, P., Callaham, D.A., and Hepler, P.K. 1994. Quantification of microtubule dynamics in living plant cells using fluorescence redistribution after photobleaching. J. Cell Sci. 107: 775-784.

Hussey, P.J., Hawkins, T.J., Igarashi, H., Kaloriti, D., and Smertenko, A. 2002. The plant cytoskeleton: Recent advances in the study of the plant microtubule-associated proteins MAP-65, MAP-190 and the Xenopus MAP215-like protein, MOR1. Plant Mol. Biol. 50: 915-924.

Igarashi, H., Orii, H., Mori, H., Shimmen, T., and Sonobe, S.
2000. Isolation of a novel $190 \mathrm{kDa}$ protein from tobacco BY-2 cells: Possible involvement in the interaction between actin filaments and microtubules. Plant Cell Physiol. 41:920-931.

Ishikawa, M., Soyano, T., Nishihama, R., and Machida, Y. 2002. The NPK1 mitogen-activated protein kinase kinase kinase contains a functional nuclear localization signal at the binding site for the NACK1 kinesin-like protein. Plant J. 32: 789-798.

Itoh, T.J., Hisanaga, S., Hosoi, T., Kishimoto, T., and Hotani, H. 1997. Phosphorylation states of microtubule-associated protein 2 (MAP2) determine the regulatory role of MAP2 in microtubule dynamics. Biochemistry 36: 12574-12582.

Jiang, W., Jimenez, G., Wells, N.J., Hope, T.J., Wahl, G.M., Hunter, T., and Fukunaga, R. 1998. PRC1: A human mitotic spindle-associated CDK substrate protein required for cytokinesis. Mol. Cell 2: 877-885.

Juang, Y.L., Huang, J., Peters, J.M., McLaughlin, M.E., Tai, C.Y., and Pellman, D. 1997. APC-mediated proteolysis of Ase1 and the morphogenesis of the mitotic spindle. Science 275: 1311-1314.

Jürgens, G. 2005. Plant cytokinesis: Fission by fusion. Trends Cell Biol. 15: 277-283.

Krysan, P.J., Jester, P.J., Gottwald, J.R., and Sussman, M.R. 2002. An Arabidopsis mitogen-activated protein kinase kinase kinase gene family encodes essential positive regulators of cytokinesis. Plant Cell 14: 1109-1120.

Kurasawa, Y., Earnshaw, W.C., Mochizuki, Y., Dohmae, N., and Todokoro, K. 2004. Essential roles of KIF4 and its binding partner PRC1 in organized central spindle midzone formation. EMBO J. 23: 3237-3248.

Mao, G., Chan, J., Calder, G., Doonan, J.H., and Lloyd, C.W. 2005. Modulated targeting of GFP-AtMAP65-1 to central spindle microtubules during division. Plant J. 43: 469-478.

Mishima, M., Kaitna, S., and Glotzer, M. 2002. Central spindle assembly and cytokinesis require a kinesin-like protein/ RhoGAP complex with microtubule bundling activity. Dev. Cell 2: 41-54.

Mollinari, C., Kleman, J.P., Jiang, W., Schoehn, G., Hunter, T., and Margolis, R.L. 2002. PRC1 is a microtubule binding and bundling protein essential to maintain the mitotic spindle midzone. J. Cell Biol. 157: 1175-1186.

Müller, S., Smertenko, A., Wagner, V., Heinrich, M., Hussey, P.J., and Hauser, M.T. 2004. The plant microtubule-associated protein AtMAP65-3/PLE is essential for cytokinetic phragmoplast function. Curr. Biol. 14: 412-417.

Nishihama, R. and Machida, Y. 2001. Expansion of the phragmoplast during plant cytokinesis: A MAPK pathway may MAP it out. Curr. Opin. Plant Biol. 4: 507-512.

Nishihama, R., Banno, H., Kawahara, E., Irie, K., and Machida, Y. 1997. Possible involvement of differential splicing in regulation of the activity of Arabidopsis ANP1 that is related to mitogen-activated protein kinase kinase kinases (MAPKKKs). Plant J. 12: 39-48.

Nishihama, R., Ishikawa, M., Araki, S., Soyano, T., Asada, T., and Machida, Y. 2001. The NPK1 mitogen-activated protein kinase kinase kinase is a regulator of cell-plate formation in plant cytokinesis. Genes \& Dev. 15: 352-363.

Nishihama, R., Soyano, T., Ishikawa, M., Araki, S., Tanaka, H., Asada, T., Irie, K., Ito, M., Terada, M., Banno, H., et al. 2002. Expansion of the cell plate in plant cytokinesis requires a kinesin-like protein/MAPKKK complex. Cell 109: 87-99.

Otegui, M.S., Verbrugghe, K.J., and Skop, A.R. 2005. Midbodies and phragmoplasts: Analogous structures involved in cytokinesis. Trends Cell Biol. 15: 404-413.

Pellman, D., Bagget, M., Tu, Y.H., Fink, G.R., and Tu, H. 1995. Two microtubule-associated proteins required for anaphase 
spindle movement in Saccharomyces cerevisiae. J. Cell Biol. 130: $1373-1385$.

Sasabe, M., Takahashi, Y., Soyano, T., Tanaka, H., Kousetsu, K., Suzuki, T., and Machida, Y. 2006. The NACK-PQR MAP kinase cascade controls plant cytokinesis. In Biotechnology in agriculture and forestry 'Tobacco BY-2 cells: A new treatise' (eds. T. Nagata, et al.). Springer, Heidelberg, Germany (in press).

Schuyler, S.C., Liu, J.Y., and Pellman, D. 2003. The molecular function of Aselp: Evidence for a MAP-dependent midzonespecific spindle matrix. J. Cell Biol. 160: 517-528.

Shelden, E. and Wadsworth, P. 1990. Interzonal microtubules are dynamic during spindle elongation. J. Cell Sci. 97: 273281.

Smertenko, A., Saleh, N., Igarashi, H., Mori, H., Hauser-Hahn, I., Jiang, C.J., Sonobe, S., Lloyd, C.W., and Hussey, P.J. 2000. A new class of microtubule-associated proteins in plants. Nat. Cell Biol. 2: 750-753.

Smertenko, A.P., Chang, H.Y., Wagner, V., Kaloriti, D., Fenyk, S., Sonobe, S., Lloyd, C., Hauser, M.T., and Hussey, P.J. 2004. The Arabidopsis microtubule-associated protein AtMAP651: Molecular analysis of its microtubule bundling activity. Plant Cell 16: 2035-2047.

Soyano, T., Nishihama, R., Morikiyo, K., Ishikawa, M., and Machida, Y. 2003. NQK1/NtMEK1 is a MAPKK that acts in the NPK1 MAPKKK-mediated MAPK cascade and is required for plant cytokinesis. Genes \& Dev. 17: 1055-1067.

Straight, A.F. and Field, C.M. 2000. Microtubules, membranes and cytokinesis. Curr. Biol. 10: R760-R770.

Strompen, G., El Kasmi, F., Richter, S., Lukowitz, W., Assaad, F.F., Jürgens, G., and Mayer, U. 2002. The Arabidopsis HINKEL gene encodes a kinesin-related protein involved in cytokinesis and is expressed in a cell cycle-dependent manner. Curr. Biol. 12: 153-158.

Tanaka, H., Ishikawa, M., Kitamura, S., Takahashi, Y., Soyano, T., Machida, C., and Machida, Y. 2004. The AtNACK1/ HINKEL and STUD/TETRASPORE/AtNACK2 genes, which encode functionally redundant kinesins, are essential for cytokinesis in Arabidopsis. Genes Cells 9: 1199-1211.

Twell, D., Park, S.K., Hawkins, T.J., Schubert, D., Schmidt, R., Smertenko, A., and Hussey, P.J. 2002. MOR1/GEM1 has an essential role in the plant-specific cytokinetic phragmoplast. Nat. Cell Biol. 4: 711-714.

Van Damme, D., Bouget, F.Y., Van Poucke, K., Inzé, D., and Geelen, D. 2004a. Molecular dissection of plant cytokinesis and phragmoplast structure: A survey of GFP-tagged proteins. Plant J. 40: 386-398.

Van Damme, D., Van Poucke, K., Boutant, E., Ritzenthaler, C., Inzé, D., and Geelen, D. 2004b. In vivo dynamics and differential microtubule-binding activities of MAP65 proteins. Plant Physiol. 136: 3956-3967.

Verbrugghe, K.J. and White, J.G. 2004. SPD-1 is required for the formation of the spindle midzone but is not essential for the completion of cytokinesis in C. elegans embryos. Curr. Biol. 14: $1755-1760$.

Vernì, F., Somma, M.P., Gunsalus, K.C., Bonaccorsi, S., Belloni, G., Goldberg, M.L., and Gatti, M. 2004. Feo, the Drosophila homolog of PRC1, is required for central-spindle formation and cytokinesis. Curr. Biol. 14: 1569-1575.

Whittington, A.T., Vugrek, O., Wei, K.J., Hasenbein, N.G., Sugimoto, K., Rashbrooke, M.C., and Wasteneys, G.O. 2001. MOR1 is essential for organizing cortical microtubules in plants. Nature 411: 610-613.

Williams, B.C., Riedy, M.F., Williams, E.V., Gatti, M., and Goldberg, M.L. 1995. The Drosophila kinesin-like protein KLP3A is a midbody component required for central spindle assem- bly and initiation of cytokinesis. J. Cell Biol. 129: 709-723. Zhu, C. and Jiang, W. 2005. Cell cycle-dependent translocation of PRC1 on the spindle by Kif4 is essential for midzone formation and cytokinesis. Proc. Natl. Acad. Sci. 102: 343-348. 


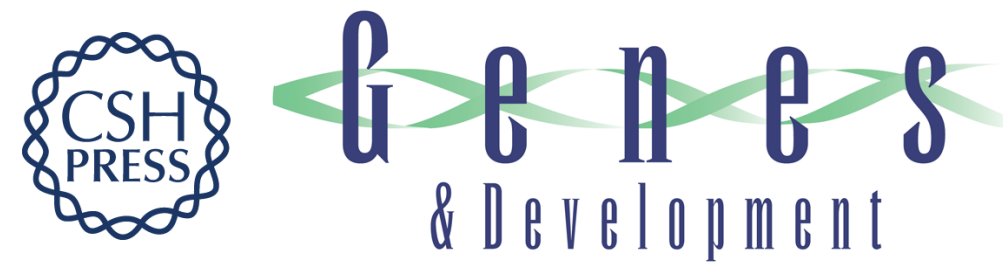

\section{Phosphorylation of NtMAP65-1 by a MAP kinase down-regulates its activity of microtubule bundling and stimulates progression of cytokinesis of tobacco cells}

Michiko Sasabe, Takashi Soyano, Yuji Takahashi, et al.

Genes Dev. 2006, 20:

Access the most recent version at doi:10.1101/gad.1408106

Supplemental Material

References

License

Email Alerting

Service
http://genesdev.cshlp.org/content/suppl/2006/04/06/gad.1408106.DC1

This article cites 48 articles, 23 of which can be accessed free at: http://genesdev.cshlp.org/content/20/8/1004.full.html\#ref-list-1

Receive free email alerts when new articles cite this article - sign up in the box at the top right corner of the article or click here.

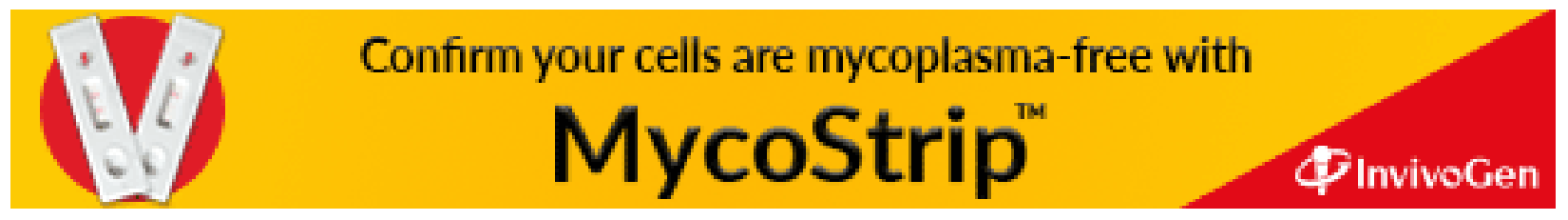

